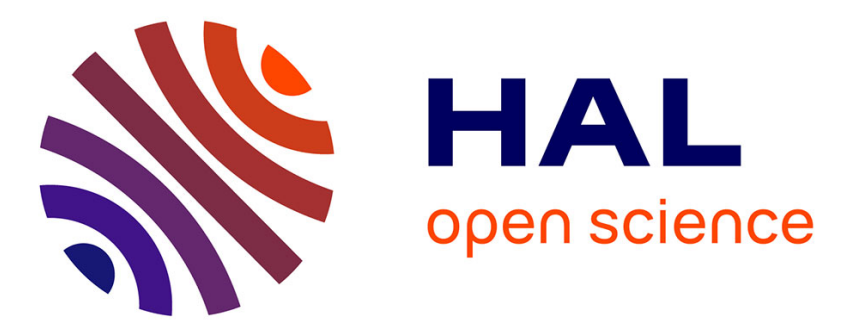

\title{
Etude des modifications temporelles des otoémissions acoustiques provoquées lors d'une stimulation acoustique controlatérale, chez l'humain normo-entendant
}

\author{
A. Giraud, E. Perrin, S. Chéry-Croze, L. Collet
}

\section{- To cite this version:}

A. Giraud, E. Perrin, S. Chéry-Croze, L. Collet. Etude des modifications temporelles des otoémissions acoustiques provoquées lors d'une stimulation acoustique controlatérale, chez l'humain normo-entendant. Journal de Physique IV Proceedings, 1994, 04 (C5), pp.C5-427-C5-430. 10.1051/jp4:1994589 . jpa-00253084

HAL Id: jpa-00253084

https://hal.science/jpa-00253084

Submitted on 1 Jan 1994

HAL is a multi-disciplinary open access archive for the deposit and dissemination of scientific research documents, whether they are published or not. The documents may come from teaching and research institutions in France or abroad, or from public or private research centers.
L'archive ouverte pluridisciplinaire HAL, est destinée au dépôt et à la diffusion de documents scientifiques de niveau recherche, publiés ou non, émanant des établissements d'enseignement et de recherche français ou étrangers, des laboratoires publics ou privés. 


\title{
Etude des modifications temporelles des otoémissions acoustiques provoquées lors d'une stimulation acoustique controlatérale, chez l'humain normo-entendant
}

\author{
A.L. GIRAUD, E. PERRIN, S. CHÉRY-CROZE et L. COLLET
}

Laboratoire de Physiologie Sensorielle, URA 1447 du CNRS, Audition et Voix, Hôpital Edouard Herriot, Pavillon U, 3 Place d'Arsonval, 69003 Lyon, France

\begin{abstract}
Résumé : In 32 subjects, the medial olivo-cochlear efferent system was activated by a contralateral acoustic stimulation (CAS) which is able to mimic the inhibitory effects of electrical stimulation of the crossed olivo-cochlear bundle. A white noise CAS of $35 \mathrm{~dB} \mathrm{SL}$ induced temporal alterations in otoacoustic emissions evoked by clicks of $63 \mathrm{~dB}$ SPL, called phase shift effect (PSE). The PSE appeared as an advance of signals in $80 \%$ of cases and occurred after a latency of about $8 \mathrm{~ms}$. Two different signal processing methods (in time and frequency domains), showed that the phase shift was mainly located on lower frequencies with a maximum around $1.5 \mathrm{kHz}$ and a lack around $4 \mathrm{kHz}$. When contralateral stimulation intensity was varying from 25 to $45 \mathrm{~dB}$ SPL, the PSE, increased linearly ; the shape of the curve allowed to rule out a middle ear reflex. On the contrary, when ipsilateral stimulation intensity was varying from 57 to $69 \mathrm{~dB}$ SPL, the PSE decreased linearly, indicating that PSE originated in inner ear mechanisms. Lastly, a significant correlation had been evidenced between the amplitude of the suppressive (inhibitory) effect of CAS, and the amplitude of the phase shift effect, allowing one to suggest that both effects may have a common physiological origin.
\end{abstract}

\section{INTRODUCTION.}

Les otoémissions acoustiques (OEA) sont des sons qui peuvent être enregistrés au niveau du conduit auditif externe. Les OEA seraient générées dans l'organe de Corti par les cellules ciliées externes (CCE), douées de propriétés contractiles actives. Les CCE, ne transmettent pas de messages auditifs, mais contribuent, gràce à leur contractilité, à amplifier l'information perçue et transmise vers les voies afférentes par les cellules ciliées internes (CCI). Les CCE reçoivent une innervation efférente provenant majoritairement de la partie médiane du complexe olivaire supérieur controlatéral. Ce système efférent médian (SEM), en modulant la contraction des CCE va donc moduler d'une part la perception au niveau des CCI et d'autre part les otoémissions acoustiques. Ainsi, il est possible d'étudier le SEM en stimulant acoustiquement une cochlée tout en enregistrant 1'activité contractile des CCE (les OEA) dans l'autre cochlée. L'utilisation de cette méthodologie a permis à de nombreux auteurs d'observer des effets de réduction de l'amplitude des OEA comparables aux effets de reduction de l'amplitude des réponses du nerf auditif lors de la stimulation électrique du faisceau olivo-cochléaire. Les études sur l'amplitude des réponses auditives ont été très nombreuses et ont servi à clarifier les modalités d'action du SEM ; en revanche, très peu d'études se sont intéressées aux autres effets d'une stimulation acoustique controlatérale. Dans cette étude nous nous proposons, en nous inspirant d'une observation de Ryan en 1991[1], de caratériser les modifications temporelles des otoémissions acoustiques provoquées par clics (OEAP), désignées par Ryan comme effet de déphasage des OEAP, lors d'une stimulation acoustique controlatérale. Nous chercherons donc à définir le sens. l'amplitude et la distribution fréquentielle de 
l'effet. Nous mettrons également en évidence l'influence de l'intensité des paramètres de stimulation ipsi et controlatéraux. Enfin nous testerons un éventuel lien statistique entre l'effet de déphasage et l'effet de réduction d'amplitude (effet suppresseur) déjà étudié par les auteurs. La caractérisation précise de l'effet de déphasage nous permettra alors de vérifier l'origine physiologique de ce déphasage et de mieux comprendre le fonctionnement du système efférent olivo-cochléaire médian.

\section{METHODES.}

\subsection{Principe}

Il s'agit de la comparaison systématique des deux OEAP enregistrées l'une en présence d'une stimulation acoustique controlatérale (CAS) et l'autre sans CAS.

\subsection{Enregistrement des OEAP}

Les otoémissions sont enregistrées, gràce à l'appareil "Otodynamic ILO88 Analyser" (Bray and Kemp, 1987[2]). Une sonde miniaturisée composée d'un haut parleur et d'un microphone, placée dans le conduit auditif externe, délivre des stimulations acoustiques et enregistre les réponses. Les stimulations sont des clics de $80 \mu \mathrm{s}$, délivrés à une cadence de 50/s. L'appareil ILO88 gère la présentation du stimulus et l'enregistrement des OEAP. Une session d'enregistrement comporte un moyennage des OEAP de 260 stimulations. L'amplitude de la réponse est exprimée en $\mathrm{dB}$. Conventionnellement, l'oreille dans laquelle sont recueillies les OEAP est qualifiée d'ipsilatérale. La stimulation acoustique controlatérale (CAS), un bruit blanc large bande $(0.05 \mathrm{à} 8 \mathrm{kHz})$, est delivrée par un audiomètre grâce à un casque.

\subsection{Expériences}

\subsection{1. population}

Les expériences ont été réalisées sur 32 sujets agés de 18 à 26 ans $(M=21$ ans), possèdant une audition normale (moins de $15 \mathrm{~dB}$ de perte sur chaque fréquence testé : $0.5,0.75,1,2,4,8 \mathrm{kHz}$ ).

\subsection{1. mise en évidence de l'effet de déphasage}

Les OEAP de 20 sujets sont enregistrées à $63 \mathrm{~dB}$ SPL de stimulation ipsilatérale sans et avec stimulation acoustique controlatérale en bruit blanc de $35 \mathrm{~dB}$ SL. Les valeurs de stimulation sont choisies de façon à ce que les enregistrements soit effectués en dessous du seuil du réflexe stapédien.

2.3.2. effets de l'intensité de stimulation ipsilatérale

Les OEAP sont enregistrées chez 12 sujets pour cinq niveaux d'intensité de clic $(57,60,63,66,69 \mathrm{~dB}$ SPL). Un enregistrement est effectué pour chaque niveau d'intensité sans et avec stimulation acoustique controlatérale à $35 \mathrm{~dB}$ SL. L'ordre de présentation des différents stimuli est aléatoire.

2.3.3. effets de l'intensité de stimulation controlaterale

Les OEAP sont enregistrées, chez 12 sujets pour une intensité de stimulation ipsilatérale fixée à $63 \mathrm{~dB}$ SPL, en absence et en présence d'une stimulation controlatérale d'intensité variable $(20,25,30,35,40$ et $45 \mathrm{~dB} \mathrm{SL}$ ), dans un ordre aléatoire.

\subsection{Analyse des modifications temporelles}

Selon la technique de Kemp, une otoémission est composée des fréquences entre 0.5 et $5.5 \mathrm{kHz}$. L'appareil procède à une analyse spectrale composée de FFT (Fast Fourier Transform) de 0.5 à $5.5 \mathrm{kHz}$.

2.4.1. mesure du décalage temporel entre les signaux

Une version récente du système ILO88 permet de mesurer le délai existant entre deux signaux. Les signaux enregistrés sans et avec stimulation controlatérale sont superposés et filtrés sur dix bandes de fréquence de 500 à $5500 \mathrm{~Hz}$. Sur chaque bande filtrée, l'un des deux signaux est déplacé pas par pas jusqu'à ce que la reproductibilité entre les deux signaux soit maximale. Les délais sont mesurés en $\mu \mathrm{s}$.

2.4.2. analyse en frequence des signaux

Le calcul de la Cohérence de Phase est utilisé pour quantifier les variations angulaires entre les deux signaux à comparer. Après analyse spectrale par FFT, la variabilité de chaque composant de Fourier des signaux est estimée par la Cohérence de Phase:

$$
\mathrm{PC}(\mathrm{f})=\left[(1 / \mathrm{n} \Sigma \cos \Phi \mathrm{i}, \mathrm{f})^{2}+(1 / \mathrm{n} \Sigma \sin \Phi \mathrm{i}, \mathrm{f})^{2}\right]^{1 / 2}
$$

où $n$ indique le nombre de spectres à comparer (ici $n=2$ ) et $\Phi \mathrm{i}, \mathrm{f}$ représente l'argument du f ième composant complexe de Fourier du i ième spectre.

\subsection{Mesure des variations d'amplitude des OEAP}

La suppression est mesurée (en $\mathrm{dB}$ ) par différence entre l'écho total (en $\mathrm{dB}$ ) de l'OEA enregistrée avec stimulation controlatérale et l'écho total de celle enregistrée sans stimulation controlatérale. 


\section{RESULTATS}

\subsection{Sens de l'effet de déphasage}

Dans $80 \%$ des cas, le déphasage a pu être observé comme une avance du signal enregistré en présence de la CAS. La figure 1 montre l'effet de déphasage ; l'effet apparaît après une latence d'environ $8 \mathrm{~ms}$. La figure 2 montre que cette latence n'est pas retrouvée lorsque le signal est filtré sur certaines bandes de fréquences; le déphasage est présent đu début à la fin de l'otoémission.

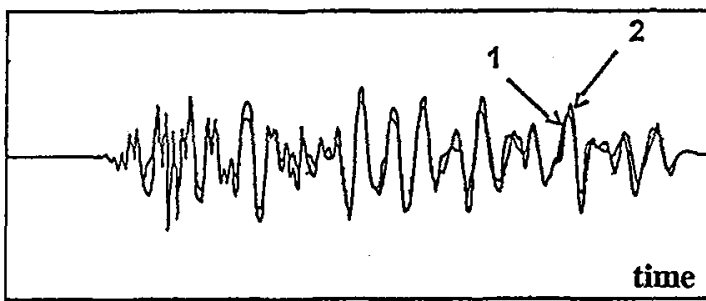

Figure 1Exemple de superposition dea deux signaux d'OEAP enregistrés avec stimulation controlaterale (sig 1), sans stimulation controlatirale (sig 2) 3.2. Distribution fréquentielle du déphasage

La distribution fréquentielle, représentée sur la figure 3, montre que l'effet concerne toutes les frequences de l'OEAP, et présente un maximum autour de $1.5 \mathrm{kHz}$. On peut noter qu'il existe une absence déphasage autour de 3.5$4 \mathrm{kHz}$.

\subsection{Amplitude de l'effet de déphasage}

Les calculs du décalage temporel entre les signaux ont montré sur 20 sujets, que le délai entre les signaux atteignait en moyenne $30 \mu \mathrm{s}$ sur les bandes les plus déphasées (1.5$2 \mathrm{kHz}$.

3.4. Effets de l'intensité de paramètres de stimulation 3.4.1. intensite de stimulation ipsilaterale

Les deux méthodes (temporelle et fréquentielle) ont montré un effet de l'intensité de la stimualtion ipsilatérale. Une corrélation négative significative est mise en évidence $(n=12 ; r=-0.86$ pour la mesure du délai et $r=-0.9$ pour l'estimation du déphasage ; $p=0.01$ ). Lorsque l'intensité croît, le déphasage diminue (figure 4).

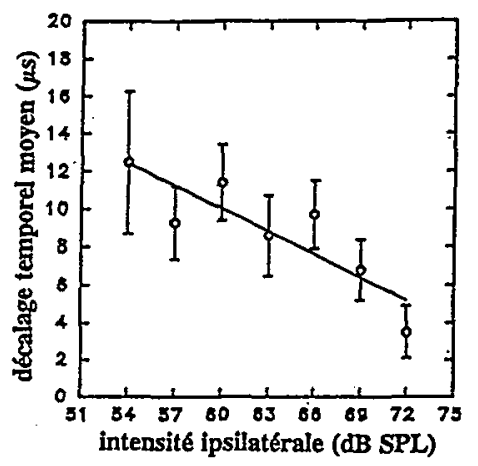

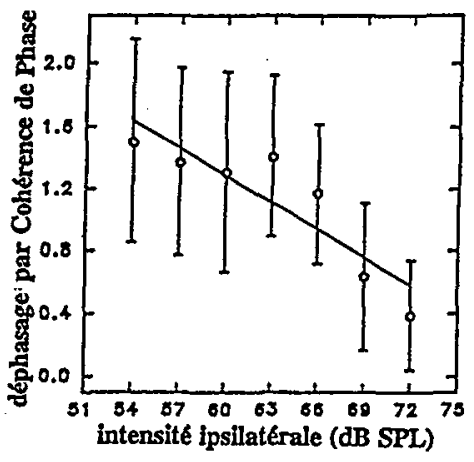

Figure 3 Distribution fréquentielle de l'eftet de déphasage. Le dephasage est estimé par différence entre la coherence de phase controle (deux OEAP enregistrés dans les mêmes conditions de stimulation, sans CAS ) et la cohérence de phase experimentale (OEAP sans versus avec CAS)

Figure 4 (gauche) Le décalage temporel ( $\mu$ s) est exprimé en fonction de l'intensité de stimulation ipsilatérale en dB SPL $n=12 ; r=-0.86 ; p=0.01$. (droite) L'estimation du déphasage est exprimé en fonction de l'intensité ipsilatérale en dB SPL $n=12 \quad r=-0.9 ; p=0.01$. 

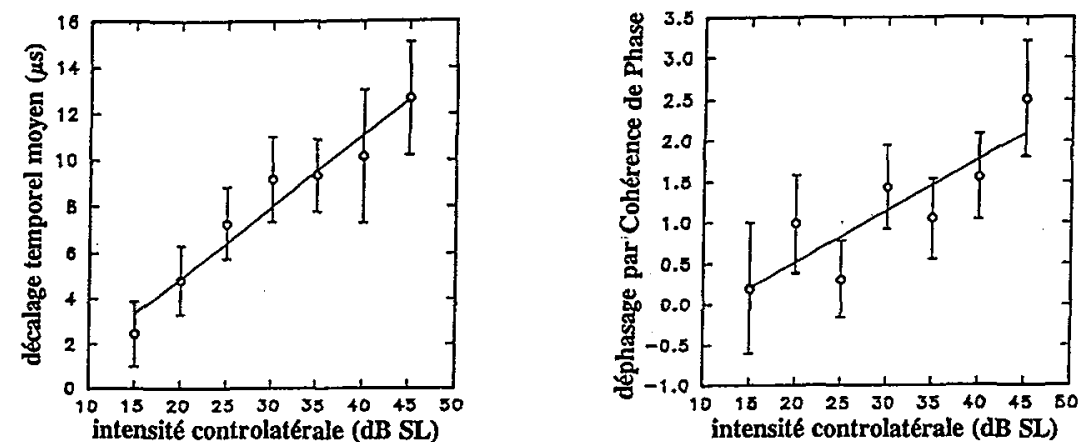

Figure 5 (gauche) Le décalage temporel en $\mu$ s est exprimé en fonction de l'intensité de stimulation controlatérale en dB SL. $n=12 ; \mathrm{r}=0.97 ; \mathrm{p}=0.01$. (droite) Estimation du déphasage est exprimé en fonction de l'intensité de stimulation controlatérale en dB SL. $n=12 ; \mathrm{r}=0.89 ; \mathrm{p}=0.01$ 3.4.2. intensité de stimulation controlatérale

Les deux méthodes mettent en évidence une corrélation positive entre intensité de la stimulation controlatérale et delai $d^{\prime} u n e$ part $(r=0.97 ; p=0.01)$ et l'estimation $d u$ déphasage, d'autre part $(\mathrm{r}=0.89 ; \mathrm{p}=0.01)$ (figure 6).

3.5. Corrélation entre effet suppresseur et déphasage

La figure 3 montre la corrélation existant entre la suppression et le décalage temporel pour chaque sujet. Les deux paramètres sont liés ; L'effet suppresseur est d'autant plus que fort le décalage temporel est important.

\section{DISCUSSION}

Cette étude met en évidence l'existence d'un déphasage des OEAP lors d'une stimulation acoustique controlatérale et confirme ainsi l'observation de Ryan (1991), tout en définissant pour la première fois les caratéristiques de cet effet.

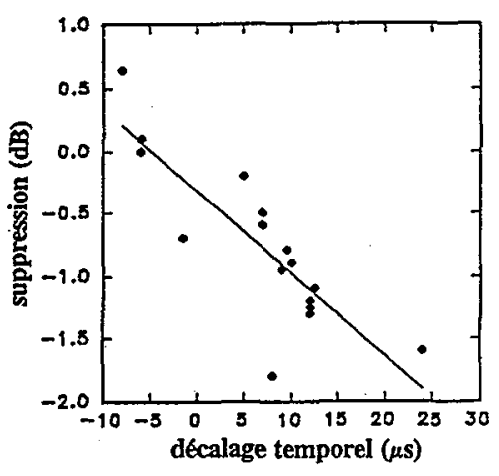

Figure 6 La suppression at le décalage temporel de chaque sujet sont représentés pat un point. La regression indique la corrélation entre les parametres. $n=16 ; r=-0.71$; $\mathrm{p}=0.01$.

Elle permet de vérifier que cet effet provient d'une activation du système efférent olivo-cochléaire médian, et non du réflexe stapédien controlatéral. En effet, les intensités utilisées sont choisies en dessous du seuil de déclanchement du réflexe ; la relation mise en évidence entre l'amplitude de l'effet de déphasage et l'intensité de stimulation controlatérale est linéaire et non sigmoidienne, montrant ainsi que l'effet étudié differe fonctionnellement d'un réflexe stapédien. D'autre part, la relation entre l'amplitude du déphasage et l'intensité de stimulation ipsilatérale indique que le déphasage est d'autant plus important que l'intensité est faible; or les CCE sont mises en jeu lors de faibles intensités de stimulation, c'est donc dans ces conditions que leurs contractions sont le plus suceptibles d'être modulées par une activation efférente. La distribution fréquentielle du déphasage indique une absence de déphasage vers $4 \mathrm{kHz}$. Cette information concorde avec une absence d'effet suppresseur observée lors d'études précédantes; il est probable que ce résultat corresponde à une baisse de la densité de l'innervation efférente vers la zone codant cette fréquence ; une telle discontinuité de l'innervation efférente ayant été observée chez l'animal, à ce niveau. L'effet de déphasage est corrélé à l'effet suppresseur, il est sensible aux mêmes paramètres de stimulation et montre une répartition fréquentielle semblable. Les auteurs ayant souvent démontré l'origine efférente de l'effet de suppression des OEA, il est très probable que les mécanismes physiologiques à l'origine des deux effets soit communs. Cette étude nuance l'action inhibitrice du SEM sur les CCE, en montrant qu'une CAS peut provoquer un départ plus précoce des OEAP, reflétant peut être un départ anticipé des messages nerveux. Il est important de poursuivre l'étude du SEM sous l'angle des modifications temporelles des réponses cochléaires afin de mieux comprendre son fonctionnement et son rôle dans la perception auditive.

\section{REFERENCES}

[1] Ryan S. , Kemp D.T. and Hinchcliffe R., British Joutnal of Audiology 25 (1992) 391-397.

[2] Bray P. and Kemp D.T., British Journal of Audiology 21 (1987) 191-204. 